九州大学学術情報リポジトリ

Kyushu University Institutional Repository

\title{
Minimax estimations
}

Mi yasawa, Koichi

Akita University

https://doi . org/10.5109/12957

出版情報 : 統計数理研究. 5 (1/2)，pp.59-76，1952-09. Research Association of Statistical Sciences

バージョン：

権利関係 : 


\title{
MINIMAX ESTIMATIONS
}

\author{
Kôichi MIYASAwa
}

\section{\$1. Introduction}

The purpose of this paper is to treat the problem of estimation from the point of view of the risk function. For that we consider the decision function as defined in our previous paper [5]. In [5] each stage of the experimentation determined by the decision function consisted of observing one and only one chance variable. But in this paper we consider the decision function in which each stage of the experimentation may consist of observing any number of chance variables and in that range of decision functions we shall find the minimax solution concerning the estimation of the mean of the normal population with the known variance. The minimax point estimation and interval estimation of the fixed length of this problem, was studied by C.R. BLYTH [2, and G. WolfowiTz [4] respectively.

But in this paper we treat the problem from the viewpoints developed in [5] for the more general case, and apply the results to the above cases as the special cases. The interesting results are those the minimax solutions of these problems are given by the nonsequential decision function, i.e. the decision functions which indicate to stop the experimentation at the first stage by taking a certain number, determined strictly by the problem, of observations.

\section{\$2. Some Lemmas}

Let $X=\left\{X_{i}\right\}(i=1,2, \cdots \cdots)$ be the sequence of random variables which are independently and identically distributed with the same distribution function $F(x)$, about which we merely know that it is an element of the given set $\Omega$ of distribution functions. Let $x=\left\{x_{i}\right\} \quad(i=1,2, \cdots \cdots)$ be the observed value of $X=\left\{X_{i}\right\}, D$ be the space of all possible terminal decisions.

We consider the decision function which consists of two sequences $\left\{\boldsymbol{B}_{n_{1}+\cdots+n_{i}}\right\}$ and $\left\{\boldsymbol{d}_{n_{1}+\cdots+n_{i}}(\boldsymbol{x})\right\}$, where $\boldsymbol{n}_{\boldsymbol{i}}$ is any positive integer $(i=1$, $2, \cdots \cdots)$.

Here $\boldsymbol{B}_{n_{1}+\cdots+n_{i}}$ is a certain subset of the space of $\left(\boldsymbol{x}_{1}, \cdots \cdots, \boldsymbol{x}_{n_{1}+\cdots+n_{i}}\right)$, $(i=1,2, \cdots \cdots)$. They are disjoint with each other, and satisfy the following relation

(1) $\sum_{i=1}^{\infty} \int_{B n_{1}+\cdots+m_{i}} p\left(F \mid x_{1}\right) \cdots \cdots p\left(F \mid x_{n_{1}+\cdots+n_{i}}\right) d x_{1} \cdots \cdots d x_{n_{1}+\cdots+n_{i}}=1$ 
for any $F \in \Omega$, where $p(F \mid x)$ is the density function of $F(x) \cdot d_{n_{1}+\cdots+m_{i}}(x)$ is the function of the first $n_{1}+\cdots+n_{i}$ coordinates of $x$ and its value is a terminal decision, i.e. an element of $D$.

Then we write

$$
\delta=\left[\left\{\boldsymbol{B}_{n_{1}+\cdots+n_{i}}\right\},\left\{\boldsymbol{d}_{n_{1}+\cdots+n_{3}}(\boldsymbol{x})\right\}\right]
$$

When the decision function (2) is adopted, the decision procedure is performed as follows.

At the first stage of the experimentation, we make observations on the chance variables $X_{1}, \cdots \cdots, X_{n_{1}}$, and obtain the observed value $x=\left(x_{1}, \cdots \cdots\right.$, $\left.x_{n_{1}}\right)$. If $x \in B_{n_{1}}$, we stop the experimentation and make the terminal decision determined by $\boldsymbol{d}_{n_{1}}(\boldsymbol{x})$.

If $x \bar{\epsilon} B_{u_{1}}$, then as the second stage of the experimentation we make observations on the chance variables $X_{n_{1}+1}, \cdots \cdots, X_{n_{1}+n_{3}}$, and obtain the observed value $\left(x_{n_{1}+1}, \cdots \cdots, x_{n_{1}+n_{2}}\right)$.

Then if $x=\left(x_{1}, \cdots \cdots, x_{n_{1}}, x_{n_{1}+1}, \cdots \cdots, x_{n_{1}+n_{2}}\right) \in B_{n_{1}+y_{2}}$, we stop the experimentation and make the terminal decision determined by $\boldsymbol{d}_{n_{1}+n_{2}}(\boldsymbol{x})$. If $x \bar{\epsilon} B_{n_{1}+n_{2}}$, we make observations on the chance variables $X_{n_{1}+n_{2}+1}, \cdots \cdots$, $X_{n_{1}+n_{2}+n_{3}}$, and proceed as above.

Let $\mathfrak{D}$ be the space of all these possible decision functions $\delta$. We assume that the cost of the experimentation is the function of the number of observations $n$ only, write it $c(n)$, and $c(n)>0$ is the monotone increasing function of $n$. Let $W(F, d) \geqq 0$ be the loss when $F$ is the true distribution function of $X_{i}$, and $d \in D$ is the terminal decision made.

Let

$$
r\left(F, d_{n}(x)\right)=c(n)+W\left(F, d_{n}(x)\right) .
$$

Then the risk of the decision function $\delta=\left[\left\{B_{n_{1}+\cdots+n_{i}}\right\},\left\{d_{n 1}+\cdots+n_{i}(x)\right\}\right]$ when $F$ is the true distribution function of $X_{i}$ is given as follows

$$
\begin{array}{r}
r(F, \grave{\delta})=\sum_{i=1}^{\infty} \int_{B_{n_{1}+\cdots+n_{i}}} r\left(F, d_{n_{1}+\cdots+n_{i}}(x)\right) p\left(F \mid x_{1}\right) \cdots \cdots \\
p\left(F \mid x_{n_{1}+\cdots+n_{i}}\right) d x_{1} \cdots \cdots d x_{n_{1}+\cdots+n_{i}}
\end{array}
$$

If $\xi$ is the a priori distribution of $F$, then the average riak of $\delta$ is given as follows, assuming that the order of integration may be changed,

$$
\begin{aligned}
& r(\xi, \delta)=\int_{\Omega} r(F, \delta) d \xi \\
& =\sum_{i=1}^{\infty} \int_{B_{n_{1}+\cdots+n_{i}}} \int_{\Omega} r\left(F, d_{n_{1}+\cdots+n_{i}}(x)\right) p\left(F \mid x_{1}\right) \cdots \cdots p\left(F \mid x_{n_{1}+\cdots+n_{i}}\right) \\
& d \xi d x_{1} \cdots \cdots d x_{n_{1}+\ldots+n_{i}} .
\end{aligned}
$$

Consequently if we put 
(6)

$$
r\left(\xi, d_{j}(x)\right)=\int_{\Omega} r\left(F, d_{j}(x)\right) p\left(F \mid x_{1}\right) \cdots \cdots p\left(F \mid x_{j}\right) d \xi
$$

for any positive integer $j$, then we can write

$$
\text { ( } 7) \quad r(\xi, \delta)=\sum_{i=1}^{\infty} \int_{B_{n_{1}+\cdots+n_{i}}} r\left(\xi, d_{n_{1}+\cdots+n_{i}}(x)\right) d x_{1} \cdots \cdots d x_{n_{1}+\cdots+n_{i}} .
$$

Let $d^{\circ}(x)$ be the function of $x=\left(x_{1}, \cdots \cdots, x_{j}\right)$ such that

$$
\begin{array}{rl}
r\left(\xi, d_{j}(x)\right)=\min _{d_{j}} & r\left(\xi, d_{j}(x)\right) \\
& \text { for any fixed } x=\left(x_{1}, \cdots \cdots, x_{j}\right),
\end{array}
$$

where $\min _{d_{j}}$ stands for the minimum with respect to the function $d_{j}(x)$ which are possible.

Let

$$
r_{j}(\xi, x)=r\left(\xi, d_{j}^{\circ}(x)\right) .
$$

Then we have the following lemma concerning the Bayes solution relative to the a priori distribution.

Lemma 1. We assume that the following (i) or $\left(\mathrm{i}^{\prime}\right)$ and (ii) hold.

(i ) $W(F, d)$ is a bounded function of $F \in \Omega$ and $d \in D$, and let $W_{0}$ be the upper bound of $W(F, d)$.

$\left(i^{\prime}\right)$ For the case $W(F, d)$ is not bounded, we consider the following situation. $\Omega$ is a one-parameter family of distribution functions $F(x, \theta)$, the $a$ priori distribution has the $\nu$-th order moment $(\nu=1,2, \cdots \cdots, m)$, and $W\left(F, d^{\circ}{ }_{j}(x)\right)$ is given as the $n$-th $(n \leqq m)$ order polynomial

$$
W\left(F, d_{j}^{\circ}(x)\right)=\sum_{i=0}^{n} f_{i j}\left(x_{1} \cdots \cdots x_{j}\right) \theta^{i}, \quad(j=1,2, \cdots \cdots),
$$

where $d^{\prime}{ }_{j}(x)$ is the function defined by (8).

For any $\varepsilon>0$, there exists $\delta>0$, such that, if

$$
\int_{c_{j}} p\left(F \mid x_{1}\right) \cdots \cdots p\left(F \mid x_{j}\right) d x_{1} \cdots \cdots d x_{j}<\delta,
$$

where $C_{j}$ is the subset of the space of $\left(x_{1}, \cdots \cdots, x_{j}\right)$, then we have

$$
\begin{aligned}
\int_{c_{j}} f_{i j}\left(x_{1} \cdots \cdots x_{j}\right) p\left(F \mid x_{1}\right) \cdots \cdots p\left(F \mid x_{j}\right) d x_{1} \cdots \cdots d x_{j}<\varepsilon \\
(i=0,1, \cdots \cdots, n ; j=1,2, \cdots \cdots) .
\end{aligned}
$$

(ii ) Let

$$
\varphi(n)=\int_{-\infty}^{\infty} r_{n}(\xi, x) d x_{n}-r_{n-1}(\xi, x), \quad(n=2,3, \cdots \cdots)
$$


The sign of $\varphi(n)$ is independent of the value of $x=\left(x_{1} \cdots \cdots x_{n-1}\right)$, but depends only on n.

Then if we can fined the positive integer $N$ such that

$$
\varphi(n)<0 \quad \text { for } 2 \leqq n \leqq N,
$$

and

$$
\varphi(n) \geqq 0 \quad \text { for } n>N,
$$

the Bayes solution $\delta_{\xi}$ relative to $\xi$, that is the decision function $\delta_{\xi}$ such that

$$
r\left(\xi, \partial_{\xi}\right)=\inf _{\delta \in \Phi} r(\xi, \delta)
$$

is given as the following decision function: we make observations on $X_{1}, \cdots \cdots, X_{N}$ and stop always the experimentation at this only one stage, and take the terminal decision determined by by $d^{\circ}{ }_{N}(x)$.

Proof. From (13) and (14), we have

$$
\begin{array}{ll}
\int_{-\infty}^{\infty} r_{i}(\xi, x) d x_{i}<r_{i-1}(\xi, x) & \text { for } i=2.3, \cdots \cdots, N, \\
\int_{-\infty}^{\infty} r_{j}(\xi, x) d x_{j} \geqq r_{j-1}(\xi, x) & \text { for } j=N+1, N+2, \cdots . .
\end{array}
$$

for any decision function

$$
\grave{o}=\left[\left\{\boldsymbol{B}_{n_{1}+\cdots+m_{q}}\right\},\left\{\boldsymbol{d}_{n_{1}+\cdots+m_{i}}(\boldsymbol{x})\right\}\right],
$$

if we take the decision function

$$
\delta^{\bullet}=\left[\left\{\boldsymbol{B}_{n_{1}+\ldots+n_{i}}\right\},\left\{\boldsymbol{d}_{{ }^{\circ}+\cdots+n_{i}}(\boldsymbol{x})\right\}\right],
$$

it is clear that

$$
r\left(\xi, \delta^{\circ}\right) \leqq r(\xi, \delta) .
$$

Consequently, if we can prove that

$$
r\left(\xi, \delta_{\xi}\right) \leqq r\left(\xi, \delta^{\circ}\right)
$$

for any decision function $\delta^{\circ}$ of the form (19), then Lemma 1 is proved. Let, for example,

$$
n_{1}+n_{2} \leqq N, \quad n_{1}+n_{2}+n_{3}>N .
$$

For the other case different from (21), the following proof can be applied in the same way. Then we have

$$
\begin{aligned}
r\left(\xi, \delta_{\xi}\right) & =\int_{-\infty}^{\infty} \ldots \int_{-\infty}^{\infty} r_{N}(\xi, x) d x_{1} \cdots \cdots d x_{N} \\
& =\int_{B_{n_{1}}} \int_{-\infty}^{\infty} \ldots \int_{-\infty}^{\infty} r_{N}(\xi, x)+\int_{C B_{n_{1}}} \int_{-\infty}^{\infty} \ldots \int_{-\infty}^{\infty} r_{N}(\xi, x) d x
\end{aligned}
$$


where $C B_{n_{1}}$ is the complement of $B_{n_{1}}$ with respect to the space of $\left(x_{1}\right.$, $\left.\cdots \cdots, x_{n_{1}}\right)$.

From (16), we have

$$
\int_{B_{n_{1}}} \int_{-\infty}^{\infty} \cdots \int_{-\infty}^{\infty} r_{N}(\xi, x) d x<\int_{B_{n_{1}}} r_{n_{1}}(\xi, x) d x .
$$

Therefore from (22) and (23), we have

$$
\int_{-\infty}^{\infty} \cdots \int_{-\infty}^{\infty} r_{N}(\xi, x) d x<\int_{B_{n_{1}}} r_{n_{1}}(\xi, x) d x+\int_{C B_{n_{1}}} \int_{-\infty}^{\infty} \ldots \int_{-\infty}^{\infty} r_{N}(\xi, x) d x .
$$

Now

$$
\begin{aligned}
\int_{C B_{n_{1}}} \int_{-\infty}^{\infty} \ldots \int_{-\infty}^{\infty} r_{N}(\xi, x) d x & =\int_{B_{n_{1}+n_{2}}} \int_{-\infty}^{\infty} \ldots \int_{-\infty}^{\infty} r_{N}(\xi, x) d x \\
& +\int_{C\left(B_{\left.n_{1}+B_{n_{1}+n_{2}}\right)} \int_{-\infty}^{\infty} \ldots \int_{-\infty}^{\infty} r_{N}(\xi, x) d x .\right.}
\end{aligned}
$$

From (16), we have

$$
\int_{B_{n_{1}+n_{2}}} \int_{-\infty}^{\infty} \ldots \int_{-\infty}^{\infty} r_{N}(\xi, x) d x \leqq \int_{B_{n_{1}}+n_{2}} r_{n_{1}+n_{2}}(\xi, x) d x .
$$

From $(24)-(26)$, we have

$$
\begin{array}{r}
\int_{-\infty}^{\infty} \ldots \int_{-\infty}^{\infty} r_{N}(\xi, x) d x<\int_{B_{n_{1}}} r_{n_{1}}(\xi, x) d x+\int_{B_{n_{1}+n_{2}}} r_{n_{1}+n_{2}}(\xi, x) d x \\
+\int_{C\left(B_{\left.n_{1}+B_{n_{1}+n_{2}}\right)}\right.} \int_{-\infty}^{\infty} \ldots \int_{-\infty}^{\infty} r_{N}(\xi, x) d x .
\end{array}
$$

From (17), we have

$$
\text { (28) } \int_{C\left(B_{n_{1}+B_{n_{1}}+n_{2}}\right)} \int_{-\infty}^{\infty} \ldots \int_{-\infty}^{\infty} r_{N}(\xi, x) d x \leqq \int_{C\left(B_{n_{1}+B_{n_{1}}+n_{2}}\right)} \int_{-\infty}^{\infty} \ldots \int_{-\infty}^{\infty} r_{n_{1}+n_{2}+n_{3}}(\xi, x) d x \text {. }
$$

Now

$$
\begin{aligned}
& \text { (29) } \int_{C\left(B_{\left.n_{1}+B_{n_{1}+n_{2}}\right)}\right.} \int_{-\infty}^{\infty} \cdots \int_{-\infty}^{\infty} r_{n_{1}+n_{2}+n_{3}}(\varphi, x) d x=\int_{\substack{B_{1}+n_{2} \\
n_{1}+n_{2}+n_{3}}} \int_{\substack{\left(n_{1}+n_{2}\right) \\
n_{1}+n_{2}+n_{3}}} r_{n_{1}+n_{2}+n_{3}}(\xi, x) d x \\
& +\int_{\substack{B_{1}+n_{2} \\
n_{1}+n_{2}+n_{3}}} \int_{\substack{B_{n_{1}+n_{2}+n_{3}}^{\left(n_{1}+n_{2}\right)} \\
n_{n_{1}+n_{3}}+B_{3}^{n_{1}+n_{2}} \\
n_{1}+n_{2}+n_{3}}} \int_{-\infty}^{\infty} \ldots \int_{-\infty}^{\infty} r_{n_{1}+n_{2}+n_{3}}(\xi, x) d x
\end{aligned}
$$

where $B_{n_{1}+n_{2}+x^{3}}^{n_{1}+n_{2}}$ denotes the set of $\left(x_{1}, \cdots \cdots, x_{n_{1}+n_{2}}\right)$ such that $\left(x_{1}, \cdots \cdots\right.$, $\left.x_{n_{1}+n_{2}+n_{3}}\right) \in B_{u_{1}+n_{2}+n_{3}}$, 
$\underset{n_{1}+n_{2}+n_{3}}{C B_{1}+n_{2}}$ is the complement cf $B_{n_{1}+n_{2}+n_{3}}^{n_{1}+n_{2}}$ relative to $C\left(B_{n_{1}}+B_{n_{1}+n_{2}}\right)$, $B_{n_{1}+n_{2}+n_{3}}^{\left(n_{1}+n_{2}\right)}$ denotes the set of $\left(x_{n_{1}+n_{2}+1}, \cdots \cdots, x_{n_{1}+n_{3}+n_{2}}\right)$ such that $\left(x_{1}, \cdots \cdots\right.$, $\left.\boldsymbol{x}_{n_{1}+n_{2}+n_{3}}\right) \in \boldsymbol{B}_{n_{1}+n_{2}+n_{3}}$,

$C B_{n_{1}+n_{2}+n_{3}}^{\left(n_{1}+n_{2}\right)}$ denotes the complement of $B_{n_{1}+n_{2}+n_{3}}^{\left(n_{1}+n_{2}\right)}$ relative to the psace of $\left(x_{n_{1}+n_{2}+1}, \cdots \cdots, x_{n_{1}+n_{2}+n_{3}}\right)$.

Then we have

(30)

$$
\int_{\substack{B_{1}+n_{2} \\ n_{1}+n_{2}+n_{3}}} \int_{\substack{\left(n_{1}+n_{2}\right) \\ n_{1}+n_{2}+n_{3}}} r_{n_{1}+n_{2}+n_{3}}(\xi, x) d x=\int_{B_{n_{1}+n_{2}+o}} r_{n_{1}+n_{2}+n_{3}}(\xi, x) d x
$$

and

$$
\begin{gathered}
\int_{B_{n_{1}+n_{2}+n_{3}}^{n_{1}+n_{2}}} \int_{\substack{n_{1}+n_{2}+n_{3} \\
\left(n_{1}+n_{2}\right)}} r_{n_{1}+n_{2}+n_{3}}(\xi, x) d x+\int_{C B_{n_{1}+n_{2}}} \int_{-\infty}^{\infty} \ldots \int_{-\infty}^{\infty} r_{n_{1}+n_{2}+n_{3}}(\xi, x) d x \\
=\int_{C\left(B n_{1}+B n_{3}+n_{3}\right.}^{\infty} \underset{\left.n_{2}+B n_{1}+n_{2}+n_{3}\right)}{r_{n_{1}+n_{2}+n_{3}}(\xi, x) d x .}
\end{gathered}
$$

Therefore from $(27)-(31)$,we have

$$
\begin{aligned}
\int_{-\infty}^{\infty} \ldots \int_{-\infty}^{\infty} r_{N} d x & <\int_{B_{n_{1}}} r_{n_{1}} d x+\int_{B_{n_{1}+n_{2}}} r_{n_{1}+n_{2}} d x \\
& +\int_{B_{n_{1}+n_{2}+n_{3}}} r_{n_{1}+n_{2}+n_{3}} d x+\int_{C\left(B_{n_{1}}+B_{n_{1}+n_{2}}+B_{\left.n_{1}+n_{2}+n_{3}\right)}\right.} r_{n_{1}+n_{3}+n_{2}} d x .
\end{aligned}
$$

Proceeding in this way, for any positive integer $k$, we have

$$
\int_{-\infty}^{\infty} \ldots \int_{-\infty}^{\infty} r_{N} d x<\sum_{i=1}^{k} \int_{B_{n_{1}+\cdots+n_{i}}} \boldsymbol{r}_{n_{1}+\cdots+n_{i}}(\xi, x) d x+\int_{C\left(\sum_{i=1}^{k} B_{\left.n_{1}+\cdots+n_{i}\right)}\right.} \boldsymbol{r}_{i, 1}+\cdots+n_{k}(\xi, x) d x .
$$

Now

$$
\begin{aligned}
& \sum_{i=k+1}^{\infty} \int_{B_{n_{1}+\cdots+n_{i}}} r_{n_{1}+\cdots+n_{i}}(\xi, x) d x \\
& =\int_{Q} \sum_{i=k+1}^{\infty} \int_{B_{n_{1}+\cdots+n_{i}}}\left\{c\left(n_{1}+\cdots \cdots+n_{i}\right)+W\left(F, d_{n_{1}+\cdots+n_{i}}^{\circ}(x)\right)\right\} \\
& \geq \int_{2} \sum_{i=k+1}^{\infty} \int_{B_{n_{1}}+\cdots+n_{i}} c\left(n_{1}+\cdots+n\right) \prod_{j=1}^{n_{1}+\cdots+n_{i}} p\left(F \mid x_{j}\right) d x_{j} d \xi
\end{aligned}
$$




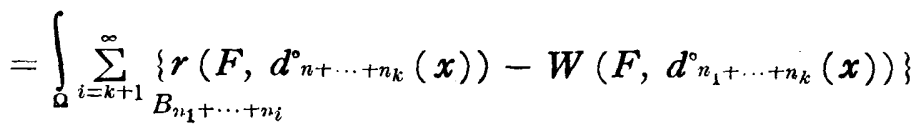

$$
\begin{aligned}
& \coprod_{j=1}^{n_{1}+\ldots+n_{i}} p\left(F \mid x_{j}\right) d x_{j} d \xi \\
& =\int_{\Omega} \int_{C\left(\sum_{i=1}^{k} B_{n_{1}}+\cdots+n_{i}\right)}\left\{\boldsymbol{r}\left(\boldsymbol{F}, \boldsymbol{d}^{\circ}{ }_{n_{1}+\cdots+n_{k}}(\boldsymbol{x})\right)-W\left(F, \boldsymbol{d}_{n_{1}+\cdots+n_{k}}^{\circ}(\boldsymbol{x})\right)\right\} \\
& \prod_{\jmath=1}^{n_{1}+\cdots+n_{k}} p\left(F \mid x_{j}\right) d x_{j} d \xi \\
& =\int_{C\left(\sum_{i=1}^{k} B_{n_{1}+\cdots+n_{i}}\right)} \boldsymbol{r}_{n_{1}+\cdots+n_{k}}(\xi, x) d x \\
& -\int_{\Omega} \int_{C\left(\sum_{i=1}^{k} B_{n_{1}}+\cdots+n_{i}\right)} W\left(F, d_{n_{1}+\cdots+n_{k}}^{\circ}(x)\right) \prod_{j=1}^{n_{1}+\cdots+n_{k}} p\left(F x_{j}\right) d x_{j} d \xi .
\end{aligned}
$$

Therefor we have

(35)

$$
\begin{aligned}
& \int_{C\left(\sum_{i=1}^{k} B_{n_{1}+\cdots+n_{i}}\right)} r_{n_{1}+\cdots+n_{k}}(\xi, x) d x \leqq \sum_{i=k+1}^{\infty} \int_{B_{n_{1}}+\cdots+n_{i}} r_{n_{1}+\cdots+n_{i}}(\xi, x) d x \\
& \quad+\int_{\Omega} \int_{C\left(\sum_{i=1}^{k} B_{n_{1}+\cdots+n_{i}}\right)} W\left(F, d_{n_{1}+\cdots+n_{k}}(x)\right) \prod_{j=1}^{n_{1}+\cdots+n_{k}} p\left(F \mid x_{j}\right) d x_{j} d \xi .
\end{aligned}
$$

Let $R_{k}$ be the second term of the right hand member of (35). Then we shall prove that, for any positive $\varepsilon$, we have

$$
R_{k} \leqq \varepsilon
$$

if $k$ is a sufficiently large positive integer.

For any $\varepsilon^{\prime}>0$, if $k$ is sufficiently large, we have from (1) that

$$
\sum_{i=k+1}^{\infty} \int_{B n_{1}+\cdots+n_{i}} p\left(F \mid x_{1}\right) \cdots \cdots p\left(F \mid x_{n_{1}+\cdots+n_{i}}\right) d x_{1} \cdots \cdots d x_{n_{1}+\cdots+n_{i}}<\varepsilon^{\prime},
$$

that is

$$
C \int_{i=1}^{k} B_{\left.n_{1}+\ldots+n_{i}\right)} \prod_{j=1}^{n_{1}+\cdots+n_{k}} p\left(F \mid x_{j}\right) d x_{j}<\varepsilon^{\prime}
$$

Consequently, if assumption (i) is sttisfied, we have 


$$
R_{k} \leqq W_{0} \int_{\Omega} \int_{C\left(\sum_{i=1}^{k} B_{\left.n_{1}+\cdots+n_{i}\right)}\right.} \coprod_{j=1}^{n_{1}+\ldots+n_{k}} p\left(F \mid x_{j}\right) d x_{j} d \xi \leqq W_{0} \varepsilon^{\prime},
$$

that is, (36) is proved,

Under the assumption $\left(i^{\prime}\right),(36)$ can also be proved in the same way. For any $\varepsilon>0$, if we take the value of $k$, such that for which (36) holds, then we have from (35) that

$$
\int_{C\left(\sum_{i=1}^{k} B_{n_{1}+\cdots+n i}\right)} \boldsymbol{r}_{n_{1}+\cdots+n_{k}}(\xi, x) d x \leqq \sum_{i=k+1}^{\infty} \int_{B_{n_{1}+\cdots+n_{i}}} \boldsymbol{r}_{n_{1}+\cdots+n_{i}}(\xi, x) d x+\varepsilon .
$$

Therefore we have

$$
\sum_{i=1}^{k} \int_{B_{n_{1}+\cdots+n_{i}}} \boldsymbol{r}_{n_{1}+\cdots+n_{i}}(\xi, x) d x+\int_{C\left(\sum_{i=1}^{k} B_{n_{1}+\cdots+n_{i}}\right)} \boldsymbol{r}_{n_{1}+\cdots+n_{k}}(\xi, x) d x<r\left(\xi, \delta^{\circ}\right)+\varepsilon
$$

From (33) and (39), we have

$$
r\left(\xi, \delta_{\xi}\right)=\int_{-\infty}^{\infty} \cdots \int_{-\infty}^{\infty} r_{N}(\xi, x) d x<r\left(\xi, \delta_{0}\right) .
$$

This shows that $\delta_{\xi}$ is a Bayes solution relative to $\xi$, and Lemma 1 is proved.

LEMMA 2.* If, for a decision function $\delta^{*}$, we can find the sequence $\left\{\xi_{k}\right\}(k=1,2, \cdots \cdots)$ of a priori distributions on such that

$$
\sup _{i^{*}} r\left(F, \delta^{*}\right)=\lim _{k \rightarrow \infty} r\left(\xi, \delta_{k}\right)
$$

where $\delta_{k}$ is the Bayes solution relative to $\xi_{k}$, then the decision function $\delta^{*}$ is minimax.

Proof. If $\delta^{*}$ is not minimax, there exists a decision function $\delta$ such that

$$
\sup r(F, \delta)<\sup _{\psi^{*}} \boldsymbol{r}\left(F, \delta^{*}\right) \text {. }
$$

Then form (41) and (42), if $k$ is sufficiently large, we have

$$
\sup _{F} \boldsymbol{r}(F, \grave{o})<\boldsymbol{r}\left(\xi_{k}, \delta_{k}\right) \text {. }
$$

On the other hand we have

$$
\sup _{k^{\prime}} \boldsymbol{r}(F, \grave{o}) \geqq \boldsymbol{r}\left(\xi_{k}, \grave{o}\right) \geq \boldsymbol{r}\left(\xi_{k}, \boldsymbol{\delta}_{k}\right) .
$$

(44) contradicts with (43), and Lemma 2 is proved.

\footnotetext{
* This lemma is essentially the same as the lemma due to E. Lehmann, cf. [2].
} 


\section{\$. Formulation of the problems}

Let $\left\{X_{k}\right\}(k=1,2, \cdots \cdots)$ be the sequence of independent chance variables, each being $N\left(\theta, \sigma^{2}\right)$, i.e., normal with the mean $\theta$ and the variance $\sigma^{2}$, where $\sigma^{2}$ is known, but $\theta$ is unknown.

The purpose of this paper is to fined the minimax estimation the mean $\theta$ of in the range $\mathfrak{D}$ of decision functions defined in $\$ 2$.

(i) General estimation.. In this case, if the decision function $o$ stops the experimentation when $n$ obsevations $x=\left(x_{1}, \cdots \cdots, x_{n}\right)$ are obtained, then its terminal decision $\boldsymbol{d}_{n k}(x)$ determines the values of two functions $L_{n k}^{1}(x)$ and $L_{n}^{2}(x),\left(L_{n}^{1} \leqq L_{n}^{2}\right)$, and indicates to estimate $\theta$ by the interval $\left(L_{n}^{1}(x)\right.$, $\left.L_{n}^{2}(x)\right)$, where $L_{n}^{1}(x)$ and $L_{n}^{2}(x)$ are functions of $x=\left(x_{1}, \cdots \cdots, x_{n}\right)$ associated with $\delta$. We assume that the cost of the experimentation is proportional to the number of observations, and the risk function is given by the following form

$$
\boldsymbol{r}\left(\theta, d_{n}(x)\right)=\mathrm{cn}+\boldsymbol{k}\left(L_{n}^{2}(x)-L_{n}^{1}(x)\right)+W\left(\theta, d_{n}(x)\right)
$$

where $c$ and $k$ are certain positive numbers, and

$$
W\left(\theta, d_{n}(x)\right)= \begin{cases}0, & \text { if } L_{n}^{1}(x) \leqq \theta \leqq L_{n}^{2}(x), \\ \left(\theta-L_{n}^{2}(x)\right)^{2}, & \text { if } \theta>L_{n}^{2}(x), \\ \left(\theta-L_{n}^{1}(x)\right)^{2}, & \text { if } \theta<L_{n}^{1}(x) .\end{cases}
$$

(ii) Estimation by an interval of the given length $2 l_{0}$. This is the special case of (i) where $d_{n}(x)$ is limited to a function such that

$$
\begin{array}{r}
L_{n}^{2}(x)-L_{n}^{1}(x)=2 l_{0}, \quad \text { for any } x=\left(x_{1}, \cdots \cdots, x_{n}\right), \\
n=1,2, \cdots \ldots
\end{array}
$$

Therefore in this case we may take $k=0$ in (45).

(iii) Point estimation. This is the special case of (ii) where $l_{0}=0$. At first we shall find the minimax solution for the general case (i), and then apply its results to cases (ii) and (iii) .

\section{\$4. General estimation.}

THEOREM 1. If the risk function is given by (45), then the minimax estimation of $\theta$ is determined as follows.

Let $\Psi_{n}(l)$ be the function of $l$ defined by

$$
\Psi_{n}(l)=\sqrt{2 \pi} \cdot k \cdot l+\int_{\sqrt{n} \cdot \sigma-1 \cdot l}^{\infty}\left(\sigma \cdot n^{-1 / 2} x-l\right)^{2} \exp \left\{-x^{2} / 2\right\} d x
$$

$h(n)$ be the function of $n$ defined by

$$
(n=1,2, \cdots \cdots)
$$

$$
\begin{aligned}
h(n)=(2 \pi)^{-1 / 2}\left\{\min _{l} \Psi_{n-1}(l)-\min _{l} \Psi_{n}(l)\right\} & \\
& (\boldsymbol{n}=2,3, \cdots \cdots) .
\end{aligned}
$$


Then $h(n)$ is a monotone decreasing function of $n$.

Consequently we candefine uniquely the positive integer $N$ as follows. If $h(2) \leqq c / 2$, then $N=2$, if $h(2)>c / 2$, then $N$ is the integer such that

$$
h(N)>c / 2 \text { and } h(N+1) \leqq c / 2 \text {. }
$$

Then the decision function $\delta_{0}$ which indicates to take observations $\left(x_{1}\right.$, $\cdots \cdots, x_{N}$ of $\left(X_{1}, \cdots \cdots, X_{N}\right)$ and stop the experimentation at this one stage and estimate $\theta$ by the interval

$$
\left(\sum_{1}^{N} x_{i} / N-l_{N}, \sum_{i}^{N} x_{i} / N+l_{N}\right)
$$

is the minimax solution of this estimation problem where is the value of $l$ such that

$$
\Psi_{n}\left(l_{n}\right)=\min _{l} \Psi_{n}(l) .
$$

Explicitely, $\min _{l} \Psi_{a}(l)$ and $l_{n}$ is given as follows.

Let $M$ be the smallest positive integer, greater than $2 \sigma^{2} / \pi k^{2}$. If $n \geqq M$, then

$$
\min _{l} \Psi_{n}(l)=(2 \pi)^{1 / 2} \sigma^{2} / 2 n, \quad l_{n}=0
$$

If $n<M$, then

$$
\min _{l} \Psi_{n}(l)=\sigma^{2} n^{-1}\left[(2 \pi)^{1 / 2}(2 \sigma)^{-1} k n^{1 / 2} s_{n}+\int_{s_{n}}^{\infty} \exp \left\{-x^{2} / 2\right\} d x\right],
$$

where $s_{n}$ the value of $s$ such that

$$
\exp \left\{-s^{2} / 2\right\}-s \int_{s}^{\infty} \exp \left\{-x_{2} / 2\right\} d x=(2 \pi)^{1 / 2}(2 \sigma)^{-1} k n^{1 / 2}
$$

and

$$
l_{n}=\sigma n^{-1 / 2} s_{n}
$$

PROOF. At first, let us assume that $\theta$ has the a priori distribution $N\left(0, b^{2}\right)$, denote it by $\xi_{b}$, and by means of of Lemma 1 we shall fined the Bayes solution $\delta_{b}$ relative to $\xi_{b}$.

In this case we have

$$
\begin{array}{r}
r\left(\varepsilon_{b}, d_{n}(x)\right)=\left(2 \pi \sigma^{2}\right)^{-n / 2}\left(2 \pi b^{2}\right)^{-1 / 2} \int_{-\infty}^{\infty} r\left(\theta, d_{n}(x)\right) \\
\quad \exp \left\{-1 / 2\left(\sum_{1}^{n}\left(x_{i}-\theta .\right)^{2} / \sigma^{2}+\theta^{2} / b^{2}\right)\right\} d \theta .
\end{array}
$$

Let us put

$$
A_{n}^{\prime}=\sum_{1}^{n} x_{i}^{2} / \sigma^{2}-b^{2}\left(\sum_{1}^{n} x^{2}\right)^{2} /\left(\sigma^{2}+n b^{2}\right) \cdot \sigma^{2}
$$




$$
\begin{aligned}
A_{n} & =\exp \left\{-A_{n}^{\prime} / 2\right\} \\
K_{n} & =\left(2 \pi \sigma^{2}\right)^{-n / 2}\left(2 \pi b^{2}\right)^{-1 / 2} \cdot A_{n} \\
a_{n} & =\sigma b\left(\sigma^{2}+n b^{2}\right)^{1 / 2} \\
Q_{n} & =-a_{n}^{2} / 2\left(\theta-\sum_{1}^{n} x_{i} / a_{n}^{2} b^{2}\right)^{2} .
\end{aligned}
$$

Then it can be shown that

$$
\begin{aligned}
& r\left(\xi_{b}, d_{n}(x)\right)=K_{n} c n(2 \pi)^{1 / 2} / a_{n}+K_{n} k(2 \pi)^{1 / 2} / a_{n} \cdot\left\{L_{n}^{2}(x)-L_{n}^{1}(x)\right\} \\
& +K_{n}\left\{\int_{L_{n}^{2}(x)}^{\infty}\left(\theta-L_{n}^{2}(x)\right)^{2} \exp Q_{n} d \theta+\int_{-\infty}^{L_{n}^{1}(x)}\left(\theta-L_{n}^{1}(x)\right)^{2} \exp Q_{n} d \theta\right\} .
\end{aligned}
$$

Therefore, from (63), in order to minimize $r\left(\xi_{u}, \boldsymbol{d}_{n}(x)\right)$ with respect to the fuction $d_{n}$ for the fixed value of $x, L_{n}^{1}(x)$ and $L_{n}^{2}(x)$ should have the following form.

$$
\begin{aligned}
& L_{n}^{1}(x)=n \bar{x} / a_{n}^{2} \sigma^{2}-l(x) \\
& L_{n}^{2}(x)=n \bar{x} / a_{n}^{2} \sigma^{2}+l(x)
\end{aligned}
$$

where $\bar{x}=\sum_{1}^{n} x_{i} / n$ and $l(x)$ is a certain function of $x=\left(x, \cdots \cdots, x_{n}\right)$. For these values of $L_{n t}^{1}(x)$ and $L_{n}^{2}(x)$, it can be shown that

$$
\begin{aligned}
r\left(\xi_{b}, d_{n}(x)\right)=2 K_{n} / a_{n k}\left[(2 \pi)^{1 / 2} c n / 2+(2 \pi)^{1 / 2} k \cdot l(x)\right. \\
\\
\left.\int_{\left.a_{n} l^{\prime} x\right)}^{\infty}\left(z / a_{n}-l(x)\right)^{2} \exp \left\{-z^{2} / 2\right\} d z\right] .
\end{aligned}
$$

Now $\Psi_{n}^{\prime \prime}(l)$ be the function of $l$ defined by

$$
\Psi_{n}^{b}(l)=(2 \pi)^{1 / 2} k \cdot l+\int_{a_{n} l}^{\infty}\left(z / a_{n}-l\right)^{2} \exp \left\{-z^{2} / 2\right\} d z
$$

Then we have

$$
r\left(\xi_{b}, d_{n}(x)\right)=2 K_{n} / a_{n n}\left[(2 \pi)^{1 / 2} c n / 2+\Psi_{n}^{b}(l(x))\right] .
$$

Consequently, for any fixed $x=\left(x_{1}, \cdots \cdots, x_{n}\right)$, the function $d_{n}$ which satisfies

$$
r\left(\xi_{b}, d_{n}^{\circ}(x)\right)=\min _{d n} r\left(\xi_{b}, d_{n}(x)\right),
$$

should determine $L_{n}^{1}(x)$ and $L_{n}^{2}(x)$ as follows

$$
\begin{aligned}
& L_{n}^{1}(x)=n \bar{x} / a_{n}^{2} \sigma^{2}-l_{n}^{b} \\
& L_{n}^{2}(x)=n \bar{x} / a_{n}^{2} \sigma^{2}+l_{n}^{b}
\end{aligned}
$$

where $l_{n}^{b}$ is the value of $l$ such that

Then, we have

$$
\Psi_{n}^{b}\left(l_{n}^{\prime \prime}\right)=\min _{l} \Psi_{n}^{b}(l) .
$$




$$
\begin{aligned}
\boldsymbol{r}_{n}\left(\xi_{n}, x\right) & =\min _{d n} \boldsymbol{r}\left(\xi_{n}, d_{n}(x)\right)=\boldsymbol{r}\left(\xi_{u}, d_{n}^{o}(x)\right) \\
& =2 K_{n} / a_{n}\left[(2 \pi)^{1 / 2} c n / 2+\Psi_{n}^{b}\left(l_{n}^{b}\right)\right] \\
& =2\left(2 \pi \sigma^{2}\right)^{-n / 2}\left(2 \pi b^{2}\right)^{-1 / 2} / a_{u}\left\{(2 \pi)^{1 / 2} c n / 2+\Psi_{n}^{b}\left(l_{n}^{b}\right)\right\} \cdot A_{n}
\end{aligned}
$$

If we put

$$
B_{n}=2\left(2 \pi \sigma^{2}\right)^{-\mu / 2}\left(2 \pi b^{2}\right)^{-1 / 2} / a_{n}\left\{(2 \pi)^{1 / 2} c n / 2+\Psi_{n}^{b}\left(l_{n}^{b}\right)\right\}
$$

then $B_{n}$ is independent of $x$ and we have

$$
r_{n}\left(\xi_{b}, x\right)=B_{n} \cdot A_{n} \text {. }
$$

Here we determine the value $l_{n}^{b}$ of $l$ which satisfies (72) and the value of $\Psi_{n}^{b}\left(l_{n}^{b}\right)$. From (67), it can be shown that

$$
\begin{aligned}
\frac{d}{d l} \Psi_{n}^{b}(l)=2 / a_{n} \cdot\left[(2 \pi)^{1 / 2} k a_{n} / 2-\exp \left\{-s^{2} / 2\right\}\right. \\
-s \int_{s}^{\infty} \exp \left\{-z^{2} / 2\right\} d z .
\end{aligned}
$$

Let the function $f(s)$ of $s$ be defined by

$$
f(s)=\exp \left\{-s^{2} / 2\right\}-s \int_{s}^{\infty} \exp \left\{-z^{2} / 2\right\} d z, \quad(s \geqq 0),
$$

then $f(s)$ is a monotone decreasing function of $s$, and $f(0)=1$. If $s_{n}$ is the value of $s$ such that

$$
(2 \pi)^{1 / 2} k a_{n} / 2=f(s)
$$

then we have

$$
l_{n}^{b}=s_{n} / a_{n} .
$$

Here, since $a_{n}$, as a function of $n$, is a monotone increasing function of $n$, we have the followings from (78).

$(2 \pi)^{1 / 2} k a_{n} / 2 \geqq 1$ is equivalent with

$$
n \geqq 2 \sigma^{2} /\left(\pi k^{2}\right)-\sigma^{2} / b^{2}
$$

Let $M^{b}$ be the smallest positive integer which satisfies $(80)$. If $n \geq M^{b}$, then we have $l_{n}^{b}=0$, and

(81) $\min _{l} \Psi_{n}^{b}(l)=\Psi_{n}^{\prime \prime}(0)=\int_{0}^{\infty} z^{2} / a_{n}^{2} \exp \left\{-z^{2} / 2\right\} d z=(2 \pi)^{1 / 2} /\left(2 a_{n}^{2}\right)$.

If $n<M^{b}$, then $l_{n}^{b}$ is given by (79), and it can be shown that

(82) $\min _{l} \Psi_{n}^{b}(l)=\Psi_{n}^{b}\left(l_{n}^{b}\right)=a_{u}^{-2}\left[(2 \pi)^{1 / 2} k a_{n} s_{n} / 2+\int_{s_{n}}^{\infty} \exp \left\{-z^{2} / 2\right\} d z\right]$

or 


$$
\begin{aligned}
=a_{n}^{-2}\left[\left(2 \pi^{1 / 2} k a_{u} s_{n} / 2+\right.\right. & s_{n}^{-1} \exp \left\{-s_{n}^{2} / 2\right\} \\
& \left.-(2 \pi)^{1 / 2} k a_{n} /\left(2 s_{n}\right)\right]
\end{aligned}
$$

Now, form (75) we have

$$
\int_{-\infty}^{\infty} r_{n}\left(\xi_{l}, x\right) d x_{n}=B_{n} \cdot \int_{-\infty}^{\infty} A_{n} d x_{n} .
$$

Here we can rewrite $A^{\prime}{ }_{n}$ as follows

$$
A_{n}^{\prime}=a_{n-1}^{2} \cdot\left(\sigma a_{n}\right)^{-2}\left\{x_{n}-b^{2} \sum_{1}^{n-1} x_{i} /\left(\sigma^{2}+(n-1) b^{2}\right)\right\}^{2}+A_{n-1}^{\prime} .
$$

Then we have

$$
\int_{-\infty}^{\infty} A_{n} d x_{n}=A_{n-1} \cdot(2 \pi)^{1 / 2} \sigma a_{n} / a_{n-1} .
$$

From (76) and (78), we have

$$
\int_{-\infty}^{\infty} r_{n}\left(\xi_{b}, x\right) d x=B_{n} \cdot A_{n-1}(2 \pi)^{1 / 2} \sigma a_{n} / a_{n-1} .
$$

Let $\varphi^{b}(n)$ be defined by

$$
\varphi^{b}(n)=\int_{-\infty}^{\infty} r_{n}\left(\xi_{b}, x\right) d x_{n}-r_{n-1}\left(\xi_{b}, x\right) .
$$

Then from (75) and (80), it can be shown that

$$
\begin{aligned}
\varphi^{b}(n)=A_{n-1}\left(2 \pi \sigma^{2}\right)^{-(n-1) / 2}\left(2 \pi b^{2}\right)^{-1 / 2} a_{n-1}^{-1} 2(2 \pi)^{1 / 2} \\
{\left[c / 2-(2 \pi)^{-1 / 2}\left\{\Psi_{n-1}^{b}\left(l_{n-1}^{b}\right)-\Psi_{n}^{b}\left(l_{n}^{b}\right)\right\}\right] . }
\end{aligned}
$$

Accordingly, the sign of $\varphi^{\prime \prime}(\boldsymbol{n})$ is independent of $x$, and dependent only of $n$. Now we define functions $h^{b}(n)$ and $g^{b}(n)$ of $n$ by the following

$$
\begin{gathered}
h^{b}(n)=(2 \pi)^{-1 / 2}\left\{\Psi_{n-1}^{b}\left(l_{n-1}^{b}\right)-\Psi_{n}^{b}\left(l_{n}^{b}\right)\right\} \\
=(2 \pi)^{-1 / 2}\left\{\min _{l} \Psi_{n-1}^{b}(l)-\min _{l} \Psi_{n}^{b}(l)\right\}, \\
g^{b}(n)=c / 2-h^{b}(n) .
\end{gathered}
$$

Then, for any fixed $x$, the sign of $\varphi^{b}(n)$ coincides with that of $g^{b}(n)$. Here we shall shown that $h^{b}(n)$ is a monotone decreasing function of $n$. For that purpose, it is sufficient to prove that the function $\Psi^{3}(n)$ of $n$ defined by

$$
\Psi^{\prime \prime}(\boldsymbol{n})=\min _{l} \Psi_{n}^{b}(\boldsymbol{l})
$$

is a convex function of $n$, that is $d^{2} \Psi^{b}(n) / d n^{2}>0$. Now it can be shown the followings.

If $n \geq M^{b}$, from (81) we have 


$$
\frac{d^{2} \Psi^{b}(n)}{d n^{2}}=(n)^{1 / 2} \sigma^{-4} a_{n}^{-6}>0
$$

If $n<M^{b}$, from (82) we have

$$
\begin{aligned}
\frac{d^{2} \Psi^{b}(n)}{d n^{2}} & =\left[2 \sigma^{4} a_{n}^{6} \int_{s_{n}}^{\infty} \exp \left\{-x^{2} / 2\right\} d x\right]^{-1} \cdot\left[\left(4 \int_{s_{n}}^{\infty} \exp \left\{-x^{2} / 2\right\} d x\right.\right. \\
& \left.\left.+s_{n} \exp \left\{-s_{n}^{2} / 2\right\}\right) \cdot \int_{s_{n}}^{\infty} \exp \left\{-x^{2} / 2\right\} d x-\exp \left\{-s_{n}^{2}\right\}\right]>0 .
\end{aligned}
$$

Therefore $h^{b}(n)$ is is a monotone decreasing function of $n$.

If $h^{b}(2) \leqq c / 2$, let $N_{b}$ be $N_{b}=2$, and

if $h^{b}(2)>c / 2$, let $N_{b}$ be the uniquely determined positive integer such that $h^{b}\left(N_{b}\right)>c / 2$, and $h^{b}\left(N_{b}+1\right) \leqq c / 2$.

Further it can be shown that the assumption ( $\left.i^{\prime}\right)$ of lemma 1 is satisfied in this problem. Consequently, from lemma 1 , we can conclude that the decision function $\delta_{,}$, which indicate to make observations $\left(x_{1}, \cdots \cdots, x_{N b}\right)$ and stop the experimentation at this one stage, and to estimate $\theta$ by the interval

$$
\left(N_{b} b^{2} \cdot \bar{x} /\left(\sigma^{2}+N_{b} b^{2}\right)-l_{N_{b}}^{b}, N_{b} b^{2} \cdot x /\left(\sigma^{2}+N_{b} b^{2}\right)+l_{N_{b}}^{b}\right)
$$

is the Bayes solution relative to $\xi_{b}$, where $\bar{x}=\sum_{1}^{N_{b}} x_{i} / N_{b}$.

Now we shall obtain the value of $r\left(\xi_{b}, \delta_{b}\right)$. From (75), we have

$$
\begin{array}{r}
r\left(\xi_{b}, \delta_{b}\right)=\int_{-\infty}^{\infty} \cdots \int_{-\infty}^{\infty} r_{N_{b}}\left(\xi_{b}, x\right) d x_{1} \cdots \cdots d x_{N_{b}} \\
=B_{N_{b}} \cdot \int_{-\infty}^{\infty} \cdots \int_{-\infty}^{\infty} A_{N_{b}} d x_{1} \cdots \cdots d x_{N_{b}} .
\end{array}
$$

Now, to obtain the value of the integral

$$
\begin{aligned}
\int_{-\infty}^{\infty} \cdots \int_{-\infty}^{\infty} A_{N} d x_{1} \cdots \cdots d x_{N} & =\int_{-\infty}^{\infty} \cdots \int_{-\infty}^{\infty} \exp \left[-\left(2 \sigma^{2}\right)^{-1}\right. \\
& \left.\left\{\sum_{1}^{N} x_{i}^{2}-b^{2}\left(\sigma^{2}+N b^{2}\right)^{-1} \cdot \sum_{1}^{N} x_{i}^{2}\right\}\right] d x_{1} \cdots \cdots d x_{N}
\end{aligned}
$$

we make the following transformation of the variables

$$
\begin{array}{rr}
x_{1}=\bar{x}+y_{1}(1 \cdot 2)^{-1 / 2}+y_{2}(2 \cdot 3)^{-1 / 2}+\cdots \cdots+y_{N-1}(\overline{N-1} \cdot N)^{-1 / 2} \\
x_{2}=\bar{x}-y_{1}(1 \cdot 2)^{-1 / 2}+y_{2}(2 \cdot 3)^{-1 / 2}+\cdots \cdots+y_{N-1}(\overline{N-1} \cdot N)^{-1 / 2} \\
x_{3}=\bar{x} & -2 y_{2}(2 \cdot 3)^{-1 / 2}+\cdots \cdots+y_{N-1}(\overline{N-1} \cdot N)^{-1 / 2} \\
x_{N}=\bar{x} & -(N-1) y_{N-1}(\overline{N-1} \cdot N)^{-1 / 2}
\end{array}
$$

Then it can be shown that we have 


$$
\begin{aligned}
\int_{-\infty}^{\infty} & \cdots \int_{-\infty}^{\infty} A_{N} d x_{1} \cdots \cdots d x_{N}=\int_{-\infty}^{\infty} \cdots \int_{-\infty}^{\infty} \exp \left[\left(2 \sigma^{2}\right)^{-1}\right. \\
& \left.\left\{\sum_{1}^{N-1} y_{i}^{2}+N \sigma^{2} \cdot \bar{x}^{2} /\left(\sigma^{2}+N b^{2}\right)\right\}\right] \\
& \sqrt{N} d y_{1} \cdots \cdots d y_{i-1} d \bar{x}=(2 \pi)^{N / 2}\left(\sigma^{2}+N b^{2}\right)^{1 / 2} \cdot \sigma^{N-1} .
\end{aligned}
$$

Therefore, from (86) and (89), we have

$$
\boldsymbol{r}\left(\xi_{b}, \delta_{l}\right)=B_{x_{b}}(2 \pi)^{x_{b} / 2} \sigma^{N_{b}-1}\left(\sigma^{2}+N_{l} \cdot b^{2}\right)^{1 / 2} .
$$

Consequently, from (74) and (90), we have

$$
\begin{array}{r}
r\left(\xi_{b^{\prime}}, \partial_{b}\right)=c N_{b}+2 k l^{b}+2(2 \pi)^{-1 / 2}(\sigma b)^{-1}\left(\sigma^{2}+N_{b} b^{2}\right)^{1 / 2} \\
\int_{l^{6}}^{\infty}\left(x-l^{\prime \prime}\right)^{2} \exp \left\{-\left(\sigma^{2}+N_{b} \cdot b^{2}\right) \cdot x^{2} / 2 \sigma^{2} b^{2}\right\} d x
\end{array}
$$

where $l^{b}$ stands for $l_{N b}^{b}$.

Now it is clear that

$$
\begin{aligned}
\lim _{l \rightarrow \infty} \Psi_{n}^{b}(l) & =\Psi_{u}(l)=(2 \pi)^{1 / 2} \cdot k \cdot l \\
& +\int_{n^{1 / 2 l / \sigma}}^{\infty}\left(n^{-1 / 2} \sigma z-l\right)^{2} \exp \left\{-z^{2} / 2\right\} d z
\end{aligned}
$$

$$
\lim _{l \rightarrow \infty} h^{\prime \prime}(n)=h(n)=(2 \pi)^{-1 / 2}\left\{\min _{l} \Psi_{n-1}(l)-\min _{l} \Psi_{n}(l)\right\} .
$$

Let $N$ be the positive intermined in Theorem 1 , then we have

$$
\lim _{b \rightarrow \infty} N_{b}=N \quad \lim _{l \rightarrow \infty} l_{x_{b}}^{b}=l_{N} \text {. }
$$

Now wa shall prove that the decision function $\partial_{0}$ which is defined in Theorem 1 is a minimax solution. We have

$$
\begin{array}{r}
r\left(\theta, \hat{o}_{0}\right)=\int_{-\infty}^{\infty} \cdots \int_{-\infty}^{\infty} r\left(\theta, d_{N}^{3}(x)\right)\left(2 \pi \sigma^{2}\right)^{-N / 2} \exp \left\{-\sum_{1}^{N}\left(x_{i}-\theta\right)^{2} / 2 \sigma^{2}\right\} \\
d x_{1} \cdots \cdots d x_{N} .
\end{array}
$$

Here we make the transformation (8). Then it can be shown that

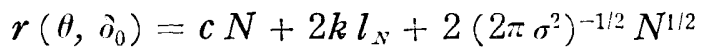

$$
\begin{aligned}
& \int_{l_{N}}^{\infty}\left(x-l_{N}\right)^{2} \exp \left\{-N x^{2} / 2 \sigma^{2}\right\} d x .
\end{aligned}
$$

From (91), (94) and (95), we have

$$
\lim _{b \rightarrow \infty} r\left(\tilde{\digamma}_{b}, \delta_{b}\right)=r\left(\theta, \hat{o}_{0}\right) \quad \text { for all } \theta \text {. }
$$

Therefore, from lemma $2, \boldsymbol{o}_{0}$ is a minimax solution of this problem. q.e.d. 


\section{§. Estimation by an interval of the given length $2 \boldsymbol{l}_{0}$.}

THEOREM 2. Let

$$
r\left(\theta, d_{n}(x)\right)=c n+W\left(\theta, d_{n}(x)\right) \quad(n=1,2, \cdots \cdots)
$$

be the risk, when one takes the sample $x==\left(x_{1}, \cdots \cdots, x_{n}\right)$, and then take the terminal decision determined by the functiond $(x)$.

Here $W\left(\theta, d_{n}(x)\right)$ is given by (46), but in this case we should have always

$$
L_{n}^{2}(x)-L_{n}^{\prime}(x)=2 l_{0} \text {. }
$$

Let

$$
\Psi_{u}\left(l_{0}\right)=\int_{u_{1} / 2_{l_{0}} / \sigma}^{\infty}\left(\sigma \cdot n^{-1 / 2} x-l_{0}\right)^{2} \exp \left\{-x^{2} / 2\right\} d x
$$

and

$$
h(\boldsymbol{n})=(2 \pi)^{-1 / 2}\left\{\Psi_{n-1}\left(l_{J}\right)-\Psi_{n}\left(l_{0}\right)\right\} .
$$

Then, $h(n)$ is a monotone decreasing function of $n$. Consequently we can define uniquely the positive integer $N$ as follows. If $h(2) \leqq c / 2$, then $N=2$, if $h(2)>c / 2$, then $N$ is the integer such that

$$
h(N)>c / 2, \quad h(N+1) \leqq c / 2 .
$$

Then the decision function $\delta_{0}$ which indicates to take observations $\left(x_{1}\right.$, $\left.\cdots \cdots, x_{N}\right)$ of $\left(X_{1}, \cdots \cdots, X_{N}\right)$ and stop the experimentation at this one stage and estimate $\theta$ by the interval

$$
\left(\sum_{1}^{N} x_{i} / N-l_{0}, \quad \sum_{1}^{N} x_{i} / N+l_{0}\right)
$$

is the minimax solution of this estimation problem.

Further, the risk function $r\left(\theta, \delta_{0}\right)$ is given as follows

$$
r\left(H, \delta_{0}\right)=c N+2(2 \pi)^{-1 / 2} N^{! / 2} \sigma \int_{l_{0}}^{\infty}\left(x-l_{3}\right)^{2} \exp \left\{-N x^{2} / 2\right\} d x .
$$

Proof. If we put $k=0$, and $l=l_{0}=$ const, in Theorem 1 , then Theorem 2 follows immediately frorn Theorem 1.

EXAMPLE. From theorem 2, it can be easily determincd the sample size $N$ which gives the minimax estimation of this problem. For example.
1) if $\sigma=1,2 l_{0}=2$,
$6 \cdot 10^{-3} \leqq c \leqq 2 \cdot 10^{-2}$, then $N=3$
2) if $\sigma=1,2 l_{0}=2$,
$2 \cdot 10^{-3} \leqq c \leqq 4 \cdot 10^{-3}$, then $N=4$
$3)$ if $\sigma=1,2 l_{0}=1 / 5$,
$6 \cdot 10^{-3} \leqq c \leqq 6 \cdot 10^{-3}$, then $N=11$
4) if $\sigma=1,2 l_{0}=10^{-1}$
$c=2 \cdot 10^{-3}$, then $N=20$. 


\section{\$6. Point estimation.}

In this case, if we take $l_{0}=0$ in Theorem 2 , then we have the following Theorem 3 immediately.

THEOREM 3. Let

$$
r\left(\theta, d_{n}(x)\right)=c n+\left(\theta-d_{n}(x)\right)^{2}
$$

be the risk, when one takes sample $x=\left(x_{1}, \cdots \cdots, x_{n}\right)$, and then estimate, the terminal decision, the value of $\theta$ by the value of the function $d_{n}(x)$. Let $N$ be the greatest positive integer smaller than

$$
\left\{1+\left(1+4 \sigma^{2} / c\right)^{1 / 2}\right\} / 2 .
$$

Then the decision function $\partial_{0}$ which indicates to take observations $\left(x_{1}\right.$, $\left.\cdots \cdots, x_{N}\right)$ of $\left(X_{1}, \cdots \cdots, X_{N}\right)$ and stop the experimentation at this one stage and estimate $\theta$ by the value

$$
\bar{x}=\sum_{1}^{N} x_{i} / N
$$

is the minimax point estimation of $\theta$.

Further, the risk function $r\left(\theta, \partial_{0}\right)$ is given as follows

$$
r\left(\theta, \partial_{0}\right)=c N+\sigma^{2} / N \text {. }
$$

Proof. In this case (99) and (100) becomes as follows

$$
\begin{gathered}
\dddot{\Psi}_{n}(0)=\sigma_{2} / n \cdot \int_{0}^{\infty} x^{2} \exp \left\{-x^{2} / 2\right\} d x=(2 \pi)^{1 / 2} \sigma^{2} /(2 n), \\
h(n)=\sigma^{2} / 2 \cdot\left\{(n-1)^{-1}-n^{-1}\right\} .
\end{gathered}
$$

Then from the cquation $c / 2-h(n)=0$, that is from

$$
c n^{2}-c n-r^{2}=0 \text {, }
$$

we have two roots of $n$

and

$$
\begin{array}{ll}
\left\{1+\left(1+4,^{2} / c\right)^{1 / 2}\right\} / 2 & (>1), \\
\left\{1-\left(1+4 r^{2} / c\right)^{1 / 2}\right\} / 2 & (<0) .
\end{array}
$$

Therefore Theorem 3 follows from Theorem 2 putting $l_{0}=0$.

EXAMPLE. In this case, the sample size $N$ which gives the minimax point estimation can be determined easily from (105).

For example we have the following table of $N$ and $r(H, \grave{o})$ for several values of $\sigma^{2}$ and $c$. 
when $\sigma^{2}=1$.

\begin{tabular}{|c|ccccc|}
\hline$c$ & 1 & $10^{-1}$ & $10^{-2}$ & $10^{-3}$ & $10^{-4}$ \\
\hline$N$ & 1 & 3 & 10 & 32 & 100 \\
\hline$r\left(\theta, \delta_{0}\right)$ & 2 & .63 & .20 & .063 & .0200 \\
\hline
\end{tabular}

when $\sigma^{2}=2$.

\begin{tabular}{|c|ccccc|}
\hline$c$ & 1 & $10^{-1}$ & $10^{-2}$ & $10^{-3}$ & $10^{-4}$ \\
\hline$N$ & 1 & 4 & 14 & 45 & 141 \\
\hline$r\left(\theta, \delta_{0}\right)$ & 3 & .90 & .28 & .089 & .0283 \\
\hline
\end{tabular}

\section{References}

(1) A. Watd, "Statistical decision functions", John Wiley and Soms, 1950.

(2) C. R. Blyth, "On minimax statistical decision problems and their admissibility", Ann. Math. Stat. Vol. 22 (1951).

(3) J. L. Hodges and E. L. LehmanN, "Some problems in minimax point estimation", Ann. Math. Stat. Vol. 21 (1950).

(4) J. Wolfowitz, "Minimax estimation of the mean of a normal distribution with known variance", Ann, Math. Stat. Vol. 21 (1950).

(5) K. Mitasawa, "On the statistical decision function I", Bull. Math. Stat. Reserch Association of Statistical Sciences. Vol. 4 (1950). 